\title{
Title: Identifying patients with hypertension using ABPM
}

British Journal of Cardiac Nursing 2014 , 9, 18-14.

Authors: Noeleen Fallon ${ }^{a}$, Gabrielle McKee ${ }^{b}$, Caroline Finn ${ }^{a}$, Nora Flynn ${ }^{a}$, Rose O’Mahony a Patricia McGeary a

Affiliations: a Cardiac Rehabilitation Department, Tallaght Hospital, Dublin 24, Ireland. ${ }^{b}$ Trinity College Dublin, School of Nursing and Midwifery, 24 D'Olier St, Dublin 2, Ireland

\section{Full details and contact of corresponding author}

Noeleen Fallon

Cardiac Rehabilitation Department, Tallaght Hospital, Dublin 24, Ireland.

Telephone 0035314143097

Fax 0035314143052

E-mail address: noeleen.fallon@amnch.ie

\begin{abstract}
:
The aim of this study was to assess the effectiveness of the systematic identification and sustained follow-up of hypertensive patients using ambulatory blood pressure monitoring (ABPM) within Phase III cardiac rehabilitation.

Methods: Patients with elevated BP (clinic measurement) on entry to cardiac rehabilitation had protocol cycles of ABPM, lifestyle education, medication changes and clinic BP monitoring, throughout cardiac rehabilitation and up to 6 months followup until BP control was achieved.
\end{abstract}

Results: Initial assessment using clinic measurement identified 30\% (129) patients with uncontrolled hypertension. Only 38\% (49) had hypertension as indicated by ABPM. These were followed up using protocol cycles up to six times. By the end $77 \%$ (99) of those originally identified as uncontrolled hypertension achieved control. 
Conclusion: This systematic identification and sustained follow up of hypertensive patients using ABPM in cardiac rehabilitation was a convenient, successful method in identifying and controlling a significant amount of uncontrolled hypertension.

Keywords: hypertension, white coat hypertension, interventions, 24hour ambulatory blood pressure monitoring Submitted August 2014, accepted November 2013 


\section{Introduction,}

Hypertension is recognized as a major modifiable risk factor for cardiovascular disease (CVD) affecting $25-44 \%$ of adults, leading to a $75 \%$ increase in CVD mortality (Mancia et al. 2007, Gu et al. 2010, Perl et al. 2012, Perk et al. 2012,).

Although the diagnosis and treatment of hypertension has played a significant part in reducing mortality and morbidity rates of CVD, levels of BP control remain poor (Perl et al. 2012, Egan et al. 2011, Roark et al. 2011). Uncontrolled BP is seen in $40-80 \%$ of the so called treated hypertensive population (Perk et al. 2012). A range of interventions, from simple to complex have been developed to help improve control of hypertension. From a hospital basis these include, self-monitoring/management, medication changes, telephone follow-up, appointment reminders, telemonitoring, ambulatory blood pressure monitoring (ABPM), hypertension clinics or more multifaceted interventions. The degree of effectiveness of the different interventions varies greatly. A recent review suggested that overall nurse or pharmacist led interventions were effective and perhaps the way forward but warranted further evaluation (Glynn et al. 2010). Assessment within these interventions, usually takes the format of a detection and sustained follow up protocol (Magid et al. 2011, Glynn et al. 2010). Secondary prevention of modifiable risk factors is the core business of CR and although BP has been seen to improve following CR (Giannuzzi et al 2008), the evidence is scant (Chatziefstratiou et al 2013 ). CR contains many core tenets of behaviour change similar to the above qualities of good BP effective interventions. It can provide the opportunity and infrastructure to implement a more formal detection and sustained follow-up protocol that would be convenient to patients with little additional resource implications. 
Routine use of $A B P M$ is recommended for the diagnoses and management of hypertension, it has been shown to result in better control of BP and more rational prescribing for BP medications, greater cost effectiveness which could result in improved quality of life for the patient (Dolan et al. 2005, Perk et al. 2012).

The aim of this study was to determine the effectiveness of a systematic identification and sustained follow-up protocol in improving hypertension control superimposed upon usual care during Phase III cardiac rehabilitation.

\section{Methods:}

To evaluate the effectiveness of the protocol, this evaluative study used a quantitative approach, a longitudinal, non-experimental, descriptive research design and retrospective convenience sampling. The study was a naturalistic design, in which the protocol was superimposed onto current practice in $\mathrm{CR}$ which utilised standard guidelines for both CR including hypertension monitoring and control (Balady et al. 2007, Hevey et al. 2007, Mancia et al. 2007, Saner \& Wood 2009, Piepoli et al. 2010, http://www.nice.org.uk/CG127/guidance),

\section{Cardiac rehabilitation format}

Eligible patients following a cardiac event such as myocardial infarction (MI), percutaneous coronary intervention $(\mathrm{PCl})$, coronary artery bypass graft (CABG), valve surgery or angina were referred to Phase III CR. Haemodynamically unstable patients such as those with severe co-morbidities, unstable angina and unstable heart failure as defined by clinical inclusion guidelines were excluded (Balady et al. 2007, Hevey et al. 2007, Saner \& Wood 2009, Piepoli et al. 2010). 
The usual standard of care in a CR program included exercise with educational talks on all aspects of CVD. This CR programme comprised of eight consecutive weeks of exercise and education. Each week three exercise with one/two education sessions, were provided by a multidisciplinary team (MDT). In line with current NICE guidelines (2011), this information included BP targets, lifestyle management with dietary advice and medication knowledge, adherence and compliance (http://www.nice.org.uk/CG127/guidance). The education was delivered by members of the MDT including dietician, physiotherapist and the nurse.

\section{Study site and population}

The study site was a large teaching hospital in Dublin, Ireland. All patients who commenced CR from January 2011-December 2012 were automatically included in the systematic identification and sustained follow-up protocol. As an effectiveness of practice study, the study required and received chairman's approval from the local relevant ethics committee.

\section{Identification and sustained follow-up protocol}

The CR nursing staff was responsible for the detection, education and follow up aspects of the BP protocol. In the week prior to the commencement of $\mathrm{CR}$ as per usual care each patient had an interview with a CR nurse and individual risk factors for CVD were assessed. BP was measured using validated DINAMAP automated BP monitors that were regularly calibrated (Baseline clinic BP). All nurses had standard training in measuring BP according to guidelines (http://www.nice.org.uk/CG127/guidance). If a patient's clinic BP was elevated, they received additional protocol education regarding 
normal BP level, lifestyle factors that influence BP and questioned regarding compliance with antihypertensive medication.

In addition to usual care one of the aims of the CR programme highlighted during the introductory talk during week one, was to control BP within target values. As per usual care during the first two weeks of the programme, the CR nurse measured clinic BP pre and post exercise class using the same BP monitors. As per protocol if measurements remained in the hypertensive range, 24 hour ABPM was carried out in week three (Baseline ABPM)

If patients' BP was elevated on the initial ABPM, a one-to-one additional session as per guidelines focusing on lifestyle management for hypertension: salt and alcohol reduction, increased exercise, increased dietary intake of fruit and vegetables, reduction in weight and compliance with medication was conducted by the nurse (http://www.nice.org.uk/CG127/guidance). At this time antihypertensive medication was altered if necessary. Clinic BP measurement continued pre and post exercise each week during CR and 24 hour ABPM was repeated during week seven, if target levels were not achieved using clinic BP measurement. If BP remained elevated with repeat $A B P M$, the patient attended an additional $C R$ clinic for education with the nurse and questioned regarding lifestyle changes and compliance to antihypertensive medication. Further alterations to current medications were made if necessary. This pattern of measuring clinic BP to initially detect hypertension and then to monitor BP response to medication, repeat $\mathrm{ABPM}$ and if $\mathrm{BP}$ remained uncontrolled, further individual education and drug titration was repeated for up to six cycles of protocol until either: BP was measured within normal range; patients were referred to external cardiology follow-up; or no further intervention was deemed appropriate by the Cardiology Registrar following review of ABPM report. 
Criteria and Data collection

In line with current hypertension guidelines thresholds, in this protocol, clinic systolic BP (SBP) of $>140 \mathrm{mmHg}$ and/or a diastolic BP (DBP) of $>90 \mathrm{mmHg}$ were defined as hypertension (http://www.nice.org.uk/CG127/guidance). High normal was defined as SBP of $130 \mathrm{mmHg}$ and DBP as $85 \mathrm{mmHg}$ (Perk et al.2012). As per hypertension guidelines ambulatory threshold differ from clinic measured values, a systolic average of $>130 \mathrm{mmHg}$ and/or a diastolic of $>80 \mathrm{mmHg}$ were defined as hypertensive (Perk et al.2012).

ABPM was measured using calibrated Spacelabs ABPM monitors and the results were analysed using Spacelabs software and then evaluated by the Cardiology Registrar (O’Brien et al. 2002).

Patients with White Coat Hypertension (WCH) were identified if clinic measurements indicated hypertension but there was no history of hypertension and BP was normal on first ABPM.

All demographic and clinical data was recorded as part of normal cardiac rehabilitation case notes and confidentially extracted for the study 6 months post cardiac rehabilitation.

\section{Analysis}

Statistical Package for Social Sciences (SPSS) version 18 was used for analysis. Clinic BP and ABPM were described in the form of mean and standard deviation. Repeated measures ANOVA was used to examine changes in clinic BP over the three time points (baseline BP, end of CR measurement and follow-up clinic appointment 
measurement) and paired samples t-tests were used to examine the changes in the ABPM over two time points (baseline ABPM, end of protocol ABPM).

\section{Results:}

Sociodemographic characteristics and screening for hypertensive patients A total of 425 patients attended cardiac rehabilitation (total population), of which $28 \%$ had a history of hypertension. Baseline clinic BP measurement revealed that 129 patients had elevated BP $(>140 / 90 \mathrm{mmHg})$ of which 10 patients had high normal BP (BP $>130 / 85 \mathrm{mmHg})$ (Perk, 2012). Patients with uncontrolled clinic BP $(n=129)$ were followed up using protocol (ABPM, education and medication titration) (Figure 1 \& 2). The socio-demographic profile of this study population is shown in Table 1.

Baseline ABPM revealed that $62 \%(n=80)$ of those with uncontrolled clinic BP had ABPM BP within the normal range (Figure 1). These included 15\% $(n=19)$, who had no previous history of hypertension and were therefore diagnosed as $\mathrm{WCH}$. Of the $38 \%(n=49)$ with uncontrolled BP, $86 \%(n=42)$ had a history of hypertension and $14 \%$ $(n=7)$ were newly diagnosed with hypertension. The uncontrolled hypertensive sample (study population) $(n=49)$ were followed up with a maximum of six cycles of protocol until their BP was measured within normal range either by clinic or ABPM or no further intervention was deemed appropriate by the cardiology registrar. Some (7\% $(n=9))$, were referred for external cardiology follow-up and $1 \%(n=1)$ were lost to followup (Figure 2, Table 2).

\section{Clinic BP changes}

At end of CR only 2 patients in the uncontrolled group was unavailable for Clinic BP, measurements. Both systolic and diastolic, for the uncontrolled hypertensive study 
sample $(n=47)$ showed a significant improvement by the end of CR (systolic BP: $F(2$, $36)=33.01, p<0.001$, diastolic BP: $F(2,36)=36.00, p=0.001)$ (Table 2). By the end of $\mathrm{CR}$ there was an average decrease in clinic systolic BP of $18.34 \mathrm{mmHg}$ and diastolic BP of $6.49 \mathrm{mmHg}$. By six months post $\mathrm{CR}$, in those available for evaluation $(n=29)$, the overall decrease in clinic systolic and clinic diastolic BP was $17.57 \mathrm{mmHg}$, and $6.45 \mathrm{mmHg}$ respectively for but only $31 \%(9 / 29)$ exhibited control (Table 2$)$.

\section{ABPM changes}

Baseline systolic and diastolic ABPM BP for the uncontrolled hypertensive study sample $(\mathrm{n}=49)$ were $137.89 \pm 9.00 \mathrm{mmHg}$ and $76.42 \pm 9.87 \mathrm{mmHg}$ respectively (Table 2$)$. At the end of protocol cycles only 35 patients were available for ABPM (10 lost to follow up and four considered not requiring further ABPM from low clinic measurements). Significant improvements were seen in both systolic $(t=3.912, p<0.001)$ and diastolic $\operatorname{ABPM}(t=2.583, p=0.014)$. The average systolic and diastolic ABPM decrease was $8.75 \mathrm{mmHg}$ and $5.65 \mathrm{mmHg}$ respectively (Table 2). A total of $54 \%(n=19)$ of the uncontrolled hypertensive study sample exhibiting control using ABPM protocol and 16 remaining uncontrolled, 10 of which had marginal values (systolic ABPM 130$139 \mathrm{mmHg}$ ). Therefore, despite 10 patients being lost to ABPM follow up, of the original population with elevated clinic BP $(n=129), 77 \%(n=99)$ patients had their BP under control using protocol. 


\section{Changes in drug use}

At ABPM baseline, in the uncontrolled hypertensive sample, the average number of hypertensive classes of drugs used was $1.97 \pm 0.72$ ranging from $0-4(n=49)$. Using clinic BP and ABPM respectively, a total of $6 \%(n=7)$ and $3 \%(n=4)$ patients were defined as having resistant hypertension - prescribed three or more classes of hypertensive drugs but BP still in hypertensive range (Mancia, 2007, Egan 2011). Over the course of the protocols, 69 drug titrations occurred, an average of 1.4 titrations per patient. The average number of hypertensive classes of drugs used in these hypertensive patients at six months was $2.13 \pm 0.76$ ranging from $1-5$. At the end of protocol using ABPM, of those still uncontrolled (SBP>130mmHg) $(n=16)$ only 2 had resistant hypertension.

\section{Discussion}

It is evident from previous studies that a multifaceted approach to hypertension control is necessary. Early diagnosis and intervention, education about lifestyle changes, medication titration and compliance, nurse or pharmacist led protocols and in particular sustained monitoring and follow-up are some of the key elements in the effective management of hypertension. This study found that a nurse-led detection and sustained follow-up intervention over six months, embedded within a cardiac rehabilitation programme, utilising additional one to one education sessions, ABPM and drug titration was successful in controlling hypertension.

In this current study using either clinic BP or ABPM measurements $31 \%$ and $54 \%$ respectively achieved control. This indicates a moderately effective intervention compared to other interventions (32\% - 63\%) (Uallachain et al. 2006, Welsh et al. 2011) 
These improvements in clinic measurements represent a $17.57 \mathrm{mmHg}$ decrease of SBP, a value on the higher side of the range of previous studies $(10.2-19.03 \mathrm{mmHg})$ (Uallachain et al. 2006, Chiu\& Wong 2010, Pladevall et al. 2010, Magid et al. 2011, Welch et al. 2011). Likewise the decrease in systolic ABPM of $8.75 \mathrm{mmHg}$ was also in line with previous measurements (Fuchs et al. 2012), but as in previous reports, was well below the range seen when using clinic measurements. A similar pattern was observed with regard to diastolic measurements: clinic measurements having a decrease of $6.45 \mathrm{mmHg}$, again within the range of previous studies $(5.0-12.2 \mathrm{mmHg})$ (Uallachain et al. 2006, Pladevall et al. 2010, Chiu \& Wong 2010, Magid et al. 2011) and diastolic ABPM values $5.65 \mathrm{mmHg}$, also in line with previous studies (Fuchs et al. 2012). These significant reductions in BP will be expected to lead to a reduction in the risk of CV events and CVD (Mancia et al. 2007, Mancia et al. 2009, Perk et al.2012, Fuchs et al. 2012), thus increasing cost effectiveness and reducing the health care burden. It has been derived that for each $10 \mathrm{mmHg}$ decrease in SBP there is a $30 \%$ decrease in CV mortality and a 40\% decrease in cerebrovascular disease (Lewington et al. 2002, Lawes et al. 2004). This intervention achieved decreases within this range therefore decreases in mortality and morbidity of this dimension would be expected. Due to the naturalistic design nature of the study we cannot distinguish which element of additional care, education, ABPM or titration of medications were the main factor in achieving these results or how much of the aforementioned changes could be attributed to lifestyle interventions instigated during the CR programme.

Sources of error that occur with clinic BP measurement remain an impediment to accurate diagnosis and treatment of hypertension but maybe reduced by adequate initial training and regular review of performance. The use of ABPM is more reliable 
and recommended in the newer prevention guidelines (Perk et al. 2012, http://www.nice.org.uk/CG127/guidance). Studies have shown that as many as 25\% of people with elevated clinic blood pressure will subsequently have normal ABPM readings (Procter-King 2011). In this study $62 \%$ of the patients were no longer diagnosed as uncontrolled hypertensive when ABPM was used. This reinforces the accuracy and necessity for using ABPM and remains one of the main arguments for using ABPM to monitor hypertensive patients.

In this study at baseline $6 \%$ of patients exhibited resistant hypertension using clinic measurements. This is below the range of previous studies $7.5 \%-16 \%$ (de las Sierra et al.2012, Daugherty et al.2012). Although there were 69 drug titrations, an average of 1.4 per patient both values were low compared to previously reported interventions (Canzanello et al. 2005). In this study, as in Crowley et al. 2011, there may have been some reluctance of doctors to medicate when BP was only mildly or moderately elevated. Medics were satisfied on occasions if the average ABPM was within high normal range $>130 / 85$ and $<140 / 90 \mathrm{mmHg}$. There may have been medical reasons for not further titrating drugs i.e. low heart rate and no room to manoeuvre with betablockers or patients presenting with side effects from medications. Therefore some of this reluctance may be well founded and represents good clinical judgement, not clinical inertia. This is especially so if clinic measurement of BP is used as the monitoring method, as found in this study when monitored using ABPM, $62 \%$ of clinic measured BP turned out to be normal. ABPM can help to assess true hypertension, resistance and clarify if further referral is required once concordance with medications is established.

There may be a need for more specific medication protocols to ensure efficient and more effective drug titration to control hypertension. Specific medication protocols 
could be managed if nurse prescribing was available within cardiac rehabilitation programmes. This would make this service cheaper - being nurse led and protocol driven. Cardiac rehabilitation is an ideal cost-effective opportunity to implement a detection, support and sustained follow-up protocol to control patients' BP. Programmes contain appropriate lifestyle education to reduce hypertension and decrease cardiovascular risk through: smoking cessation, weight reduction, alcohol consumption moderation, physical activity, increasing vegetable and fruit intake, decreasing saturated and total fats and reduction in salt intake (Mancia et al. 2007). The multidisciplinary nature of cardiac rehabilitation enables the provision of such education and support in order for patients to understand treatment targets and adjust their lifestyle (Perl et al. 2012).

\section{Conclusion:}

The enormity of the challenge of hypertension control demands a coordinated approach. The proportion of patients that achieved BP control compared to other interventions was good. Utilising available resources within cardiac rehabilitation may prove a cost effective method of targeting hypertension, however there was room for further effectiveness through further drug titrations. 


\section{Implications for Practice:}

- Diagnose hypertension \& white coat hypertension with ABPM

- Sustained follow-up protocols are needed to control BP

- Know and utilise guidelines and targets for goal setting

- Holistic approach by a multidisciplinary team is an effective way to educate patients and effect control.

\section{Acknowledgments}

The authors wish to acknowledge the contribution of data from individual patients who attended cardiac rehabilitation. They also wish to acknowledge the support from colleagues who read the paper and offered constructive criticism. 
Table 1: Patients demographics $(n=129)$

\begin{tabular}{|c|c|c|}
\hline Age & & $61.87 \pm 11.59$ \\
\hline Gender ( Male) & & $76.9 \%(97)$ \\
\hline Marital status (married) & & $92 \%(115)$ \\
\hline BMI & & $29.97 \pm 4.65$ \\
\hline Waist Circumference & & $101.1 \pm 11.3$ \\
\hline Smoking status & Never & $40.5 \%(51)$ \\
\hline & Ex smoker & $52.4 \%(66)$ \\
\hline & Current & $7.1 \%(9)$ \\
\hline Alcohol (units of alcohol co & insumed per week) & $8.62 \pm 10.73$ \\
\hline Diabetes & & $15 \%(19)$ \\
\hline Lipid profile & Low density lipoproteins & $1.9 \pm 0.62$ \\
\hline & High density lipoproteins & $1.16 \pm 0.34$ \\
\hline & Total cholesterol & $3.71 \pm 0.85$ \\
\hline & Triglycerides & $1.53 \pm 1.51$ \\
\hline History of hypertension & & $81.6 \%(102)$ \\
\hline Hypertension medication & Beta blockers & $76.9 \%(97)$ \\
\hline & ACE inhibitors & $56.3 \%(71)$ \\
\hline & Angiotensin receptor blocker & $17.5 \%(22)$ \\
\hline & Calcium channel blockers & $19.8 \%(25)$ \\
\hline & Diuretics & $3.2 \%(4)$ \\
\hline Treatment & Percutaneous coronary intervention & $48.4 \%(61)$ \\
\hline & Coronary artery bypass graft & $30.1 \%(38)$ \\
\hline & Medically managed CAD & $21.4 \%(27)$ \\
\hline Diagnosis & Coronary artery disease (CAD) & $52.8 \%(66)$ \\
\hline & Non ST elevation myocardial infarction & $17.6 \%(21)$ \\
\hline & ST elevation myocardial infarction & $12.8 \%(16)$ \\
\hline & Angina & $6.4 \%(8)$ \\
\hline & Valve Surgery & $4.8 \%(6)$ \\
\hline & Other & $5.6 \%(7)$ \\
\hline Attendance at CR (\% of tot & al course attended) & $83.08 \pm 13.54$ \\
\hline
\end{tabular}


Table 2: Changes in BP over time in the uncontrolled group

\begin{tabular}{|l|l|l|l|}
\hline & $\begin{array}{l}\text { Baseline BP } \\
\text { measurement } \\
(n=49)\end{array}$ & $\begin{array}{l}\text { End of CR BP } \\
\text { measurement } \\
(n=47)\end{array}$ & Final BP measurement \\
\hline ABPM systolic & $137.89 \pm 9.00$ & & $129.14 \pm 11.19(n=29)$ \\
\hline ABPM diastolic & $76.42 \pm 9.87$ & & $70.77 \pm 10.16(n=29)$ \\
\hline Clinic systolic & $162.65 \pm 17.06$ & $144.31 \pm 14.89$ & $145.08 \pm 15.3(n=35)$ \\
\hline Clinic diastolic & $84.40 \pm 11.97$ & $77.91 \pm 9.50$ & $77.95 \pm 8.94(n=35)$ \\
\hline
\end{tabular}


Figure 1: Flow chart indicating patients BP diagnosis

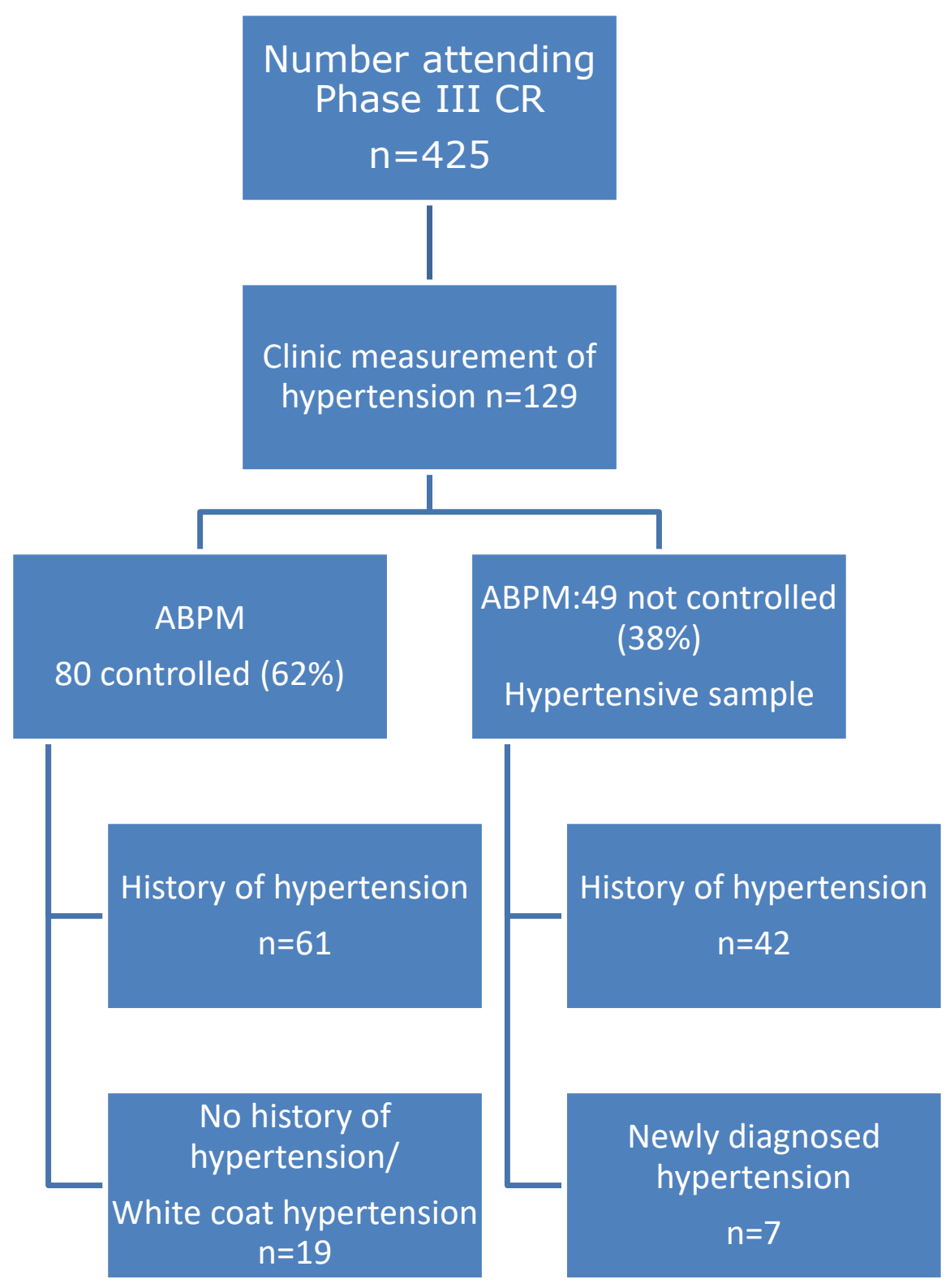


Figure 2 Follow up of Hypertensive patients using Protocol

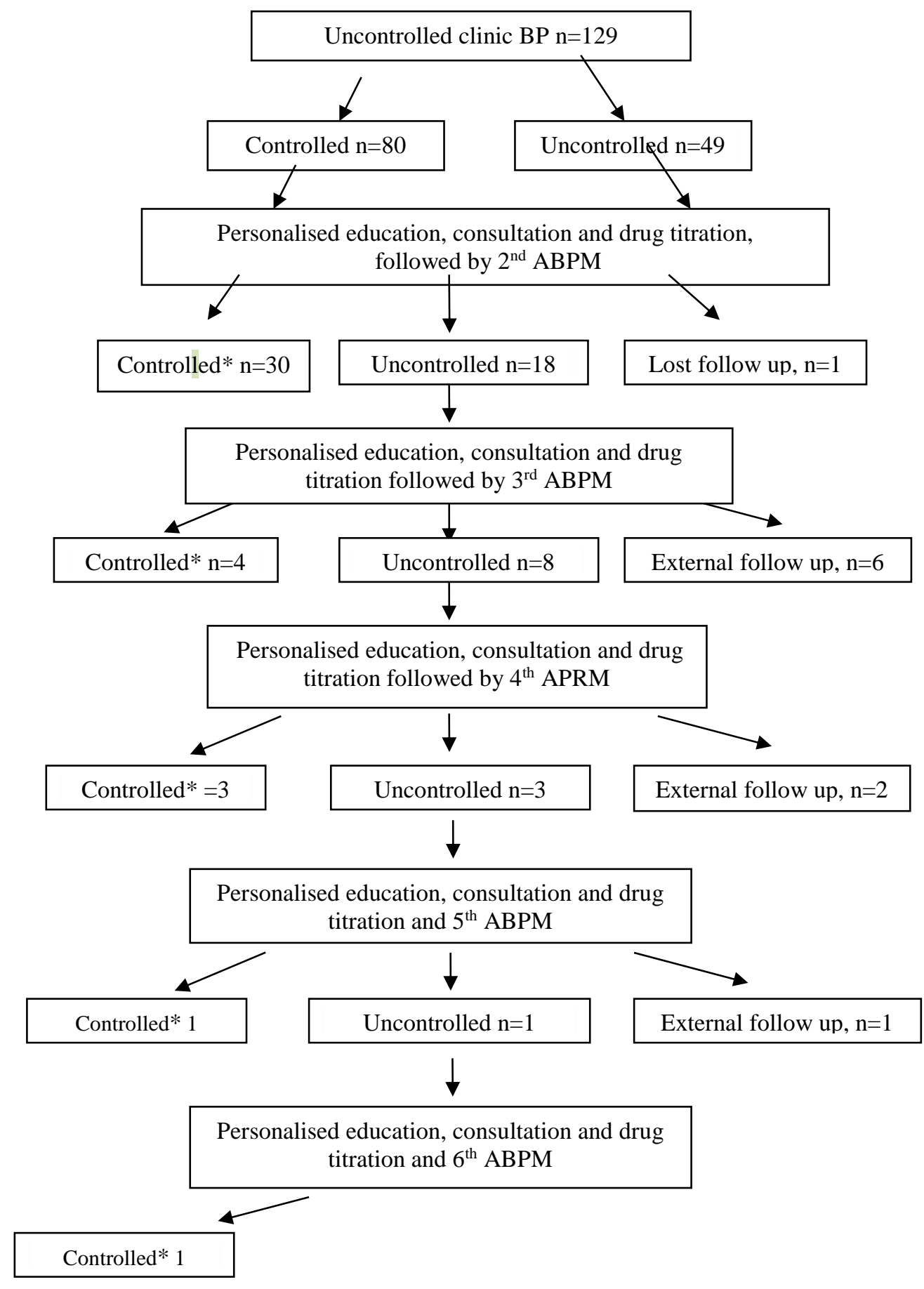

${ }^{*}$ Controlled:indicates patients whose ABPM fell within the target ranges or no further intervention was deemed appropriate by the Cardiology Registrar 


\section{References}

Balady GJ, Williams MA, Ades PA, Bittner V, Comoss P, Foody JM, et al. (2007) Core components of cardiac rehabilitation/secondary prevention programs: 2007 update: a scientific statement from the American Heart Association Exercise, Cardiac Rehabilitation, and Prevention Committee, the Council on Clinical Cardiology; the Councils on Cardiovascular Nursing, Epidemiology and Prevention, and Nutrition, Physical Activity, and Metabolism; and the American Association of Cardiovascular and Pulmonary Rehabilitation. Circulation 115:2675-82

Chatziefstratiou AA, Giakoumidakis K, Brokalaki H. Cardiac rehabilitation outcomes: modifiable risk factors. British Journal of Nursing. 2013;22(4):200-7.

Chiu CW, Wong FKY (2010) Effects of 8 weeks sustained follow-up after a nurse consultation on hypertension: A randomised trial. Int J Nurs Stud 47:1374-82

Daugherty SL, Powers JD, Magid DJ, Tavel HM, Masoudi FA, Margolis KL, et al. (2012) Incidence and prognosis of resistant hypertension in hypertensive patients. Circulation 125:1635-42

Dolan E, Stanton A, Thijs L, Hinedi K, Atkins N, McClory S, et al.(2005) Superiority of ambulatory over clinic blood pressure measurement in predicting mortality: the Dublin outcome study. Hypertension 46:156-61

Egan BM, Zhao Y, Axon RN, Brzezinski WA, Ferdinand KC. (2011) Uncontrolled and apparent treatment resistant hypertension in the United States, 1988 to 2008. Circulation 124:1046-58

Giannuzzi P, Temporelli PL, Marchioli R et al (2008) Global secondary prevention strategies to limit event recurrence after myocardial infarction: results of the GOSPEL study, a multicenter, randomized controlled trial from the Italian Cardiac Rehabilitation Network. Arch Intern Med 168(2): 2194-2204

Glynn L, Murphy A, Smith S, Schroeder KTF. (2010) Interventions used to improve control of blood pressure in patients with hypertension. Cochrane Database Syst Review.CD005182

Gu Q, Dillon CF, Burt VL, Gillum RF. (2010) Association of hypertension treatment and control with all-cause and cardiovascular disease mortality among US adults with hypertension. Am J Hypertens 23:38-45

Head GA, McGrath BP, Mihailidou AS, Nelson MR, Schlaich MP, Stowasser M, et al. (2012) Ambulatory blood pressure monitoring in Australia: 2011 consensus position statement. $J$ Hypertens 30:253-66

Hevey DCK, Kingston C, Fallon N, Horgan JH ( 2007) Irish Ass of Cardiac Rehabilitation Guidelines 2007 . Retrieved 14 $4^{\text {th }}$ May 2013 http://www.iacr.info/page11.htm 
Magid DJ, Ho PM, Olson KL, Brand DW, Welch LK, Snow KE, et al. (2011) A Multimodal Blood Pressure Control Intervention in 3 Healthcare Systems. Am J Man Care 17:e96-e103

Mancia G, De Backer G, Dominiczak A, Cifkova R, Fagard R, Germano G, et al (2007) Guidelines for the management of arterial hypertension: The Task Force for the Management of Arterial Hypertension of the European Society of Hypertension (ESH) and of the European Society of Cardiology (ESC). Eur Heart J 28:1462-536

Mancia G, Laurent S, Agabiti-Rosei E, Ambrosioni E, Burnier M, Caulfield MJ, et al.( 2009)Reappraisal of European guidelines on hypertension management: a European Society of Hypertension Task Force document. Blood Press 18:308-47

O'Brien E, Atkins N, Stergiou G, Karpettas N, Parati G, Asmar R, et al.( 2010) European Society of Hypertension International Protocol revision 2010 for the validation of blood pressure measuring devices in adults. Blood Press Monit 15:23-38

Perk J, De Backer G, Gohlke H, Graham I, Reiner Z, Verschuren WM, et al. (2012) European Guidelines on Cardiovascular Disease Prevention in Clinical Practice (Version 2012) : The Fifth Joint Task Force of the European Society of Cardiology and Other Societies on Cardiovascular Disease Prevention in Clinical Practice (Constituted by Representatives of Nine Societies and by Invited Experts). Eur Heart J 33:1635-1701

Perl S, Riegelnik V, Mrak P, Ederer H, Rakovac I, Beck P, et al.(2011) Effects of a multifaceted educational program on blood pressure and cardiovascular risk in hypertensive patients: the Austrian herz.leben project. $J$ Hypertens 29:2024-30

Pladevall M, Brotons C, Gabriel R, Arnau A, Suarez C, Marquez E, et al. (2010) Multicenter clusterrandomized trial of a multifactorial intervention to improve antihypertensive medication adherence and blood pressure control among patients at high cardiovascular risk (the COM99 study). Circulation 122:1183-91

Piepoli MF, Corra U, Benzer W, Bjarnason-Wehrens B, Dendale P, Gaita D, et al.( 2010) Secondary prevention through cardiac rehabilitation: from knowledge to implementation. A position paper from the Cardiac Rehabilitation Section of the European Association of Cardiovascular Prevention and Rehabilitation. Eur J Cardiovasc Prev Rehabil 17:1-17

Roark RF, Shah BR, Udayakumar K, Peterson ED.(2011)The need for transformative innovation in hypertension management. Am Heart J 162:405-11

Saner $\mathrm{H}$, Wood D. Practical organisation of preventive cardiology programmes: integrating prevention and rehabilitation (2009) Eur J Cardiovasc Prev Rehabil 16 Suppl 2:S37-42 
de la Sierra A, Segura J, Banegas JR, Gorostidi M, de la Cruz JJ, Armario P, et al. (2011) Clinical features of 8295 patients with resistant hypertension classified on the basis of ambulatory blood pressure monitoring. Hypertension 57:898-902

Uallachain GN, Murphy G, Avalos G (2006). The RAMBLER study: the role of ambulatory blood pressure measurement in routine clinical practice: a cross-sectional study. Ir Med J 99:276-9.

Verdecchia P, Angeli F. (2005) How can we use the results of ambulatory blood pressure monitoring in clinical practice? Hypertension 46:25-6

Welch LK, Olson KL, Snow KE, Pointer L, Lambert-Kerzner A, Havranek EP, et al. (2011) Systolic Blood Pressure Control After Participation in a Hypertension Intervention Study. Am J Manag Care 17:473-8 